\title{
Correction: Vol. 25, No. 3
}

Figures 2 and 4 were incomplete in Donor-Derived Genotype 4 Hepatitis E Virus Infection, Hong Kong, China, 2018 (S. Sridhar et al.). The corrected figures are reproduced here, and the article has been corrected online (https://wwwnc.cdc. gov/eid/article/25/3/18-1563_article).

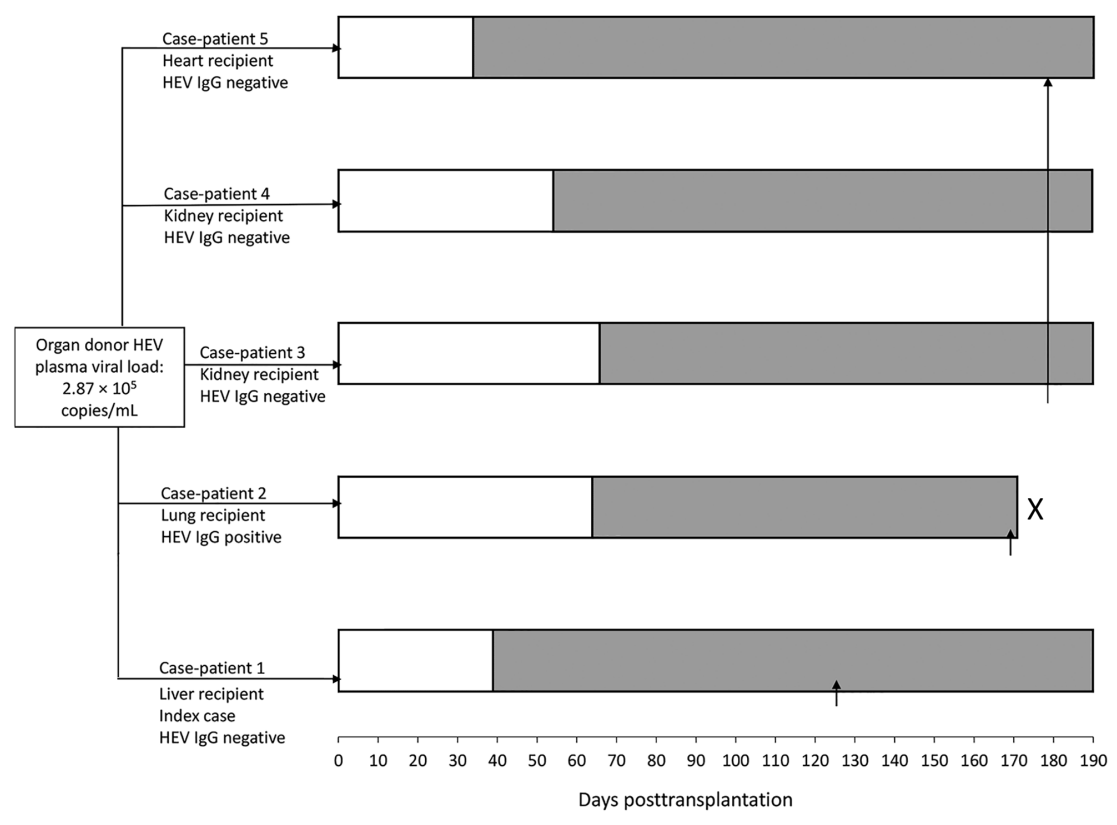

Figure 2. Timeline of outbreak in study of donorderived genotype $4 \mathrm{HEV}$ infection, Hong Kong, China, 2018, showing baseline HEV IgG status of each organ recipient. White bars indicate incubation period during which liver function test results were within reference range. Gray bars indicate timeline of alanine aminotransferase derangement after transplantation. $\mathrm{X}$ indicates patient death. Vertical arrows $(\uparrow)$ indicate time of hepatitis $\mathrm{E}$ diagnosis. HEV, hepatitis $E$ virus.
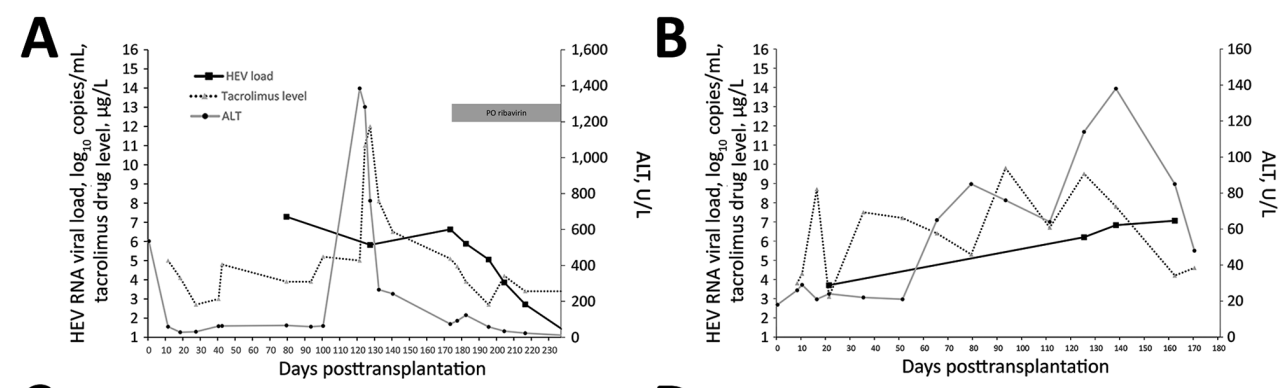

C
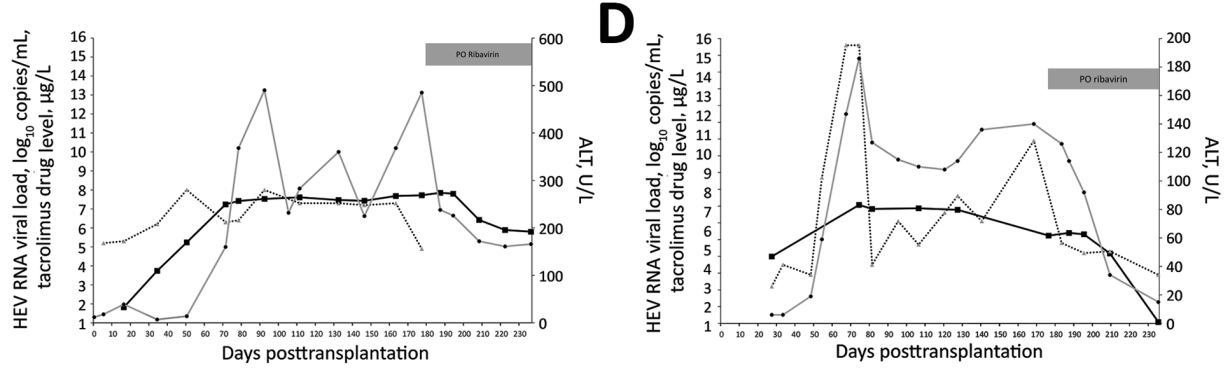

E

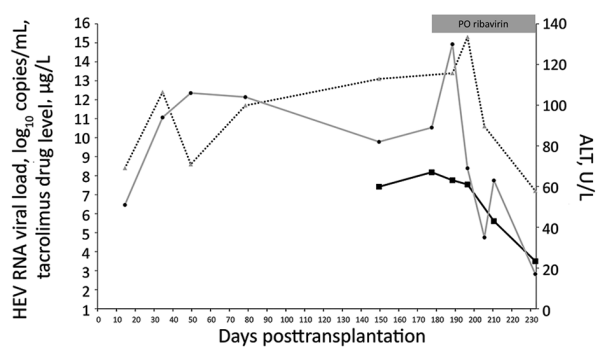

Figure 4. Kinetics of liver function test (ALT) results, tacrolimus levels, and plasma HEV RNA load with relation to ribavirin therapy. A) Casepatient 1; B) case-patient 2; C) case-patient 3; D) case-patient 4 ; E) case-patient 5 . Date for casepatients 1, 3, 4, and 5 were updated up to week 8 of ribavirin treatment. Horizontal gray bars indicate when patient began taking oral ribavirin. ALT, alanine aminotransferase; HEV, hepatitis E virus. 